Mathematical Models and Methods in Applied Sciences

Vol. 25, No. 14 (2015) 2795-2800

(C) World Scientific Publishing Company

DOI: $10.1142 / \mathrm{S} 0218202515990018$

\title{
Author index Volume 25
}

Agnelli, J. P., Colasuonno, F. \& Knopoff, D., A kinetic theory approach to the dynamics of crowd evacuation from bounded domains

Agostiniani, V., Blass, T. \& Koumatos, K., From nonlinear to linearized elasticity via $\Gamma$-convergence: The case of multiwell energies satisfying weak coercivity conditions

Aguillon, N., Riemann problem for a particle-fluid coupling

Ambrosi, D., see Penta

Andreianov, B., Bendahmane, M., Quarteroni, A. \& Ruiz-Baier, R., Solvability analysis and numerical approximation of linearized cardiac electromechanics

Aoki, K., Pulvirenti, M., Simonella, S. \& Tsuji, T., Backward clusters, hierarchy and wild sums for a hard sphere system in a low-density regime

Arita, C. \& Schadschneider, A., Exclusive queueing processes and their application to traffic systems

Bae, H.-O., Ha, S.-Y., Kim, Y., Lee, S.-H., Lim, H. \& Yoo, J., A mathematical model for volatility flocking with a regime switching mechanism in a stock market

Bae, M., Duan, B. \& Xie, C., Two-dimensional subsonic flows with selfgravitation in bounded domain
Barbatis, G., Stratis, I. G. \& Yannacopoulos, A. N., Homogenization of random elliptic systems with an application to Maxwell's equations

25 (2015) 1365

Bassetti, F. \& Toscani, G., Mean field dynamics of interaction processes with duplication, loss and copy

Bazilevs, Y., Korobenko, A., Yan, J., Pal, A., Gohari, $25(2015) 1 \quad$ S. M. I. \& Sarkar, S. ALE-VMS formulation for stratified turbulent incompressible flows with applications

$25(2015) 79$

Bazilevs, Y., Takizawa, K. \& Tezduyar, T. E., New directions and challenging computations in fluid dynamics modeling with stabilized and multiscale methods

Beirão da Veiga, L., Hughes, T. J. R., Kiendl, J., Lovadina, C., Niiranen, J., Reali, A. \& Speleers, H., A locking-free model for Reissner-Mindlin plates: Analysis and isogeometric implementation via NURBS and triangular NURPS

25 (2015) 401

Bellomo, N. \& Brezzi, F., Traffic, crowds, and dynamics of self-organized particles: New trends and challenges

Bellomo, N. \& Gibelli, L., Toward a mathematical theory of behavioralsocial dynamics for pedestrian crowds

Bellomo, N., Bellouquid, A., Tao, Y. \& Winkler, M., Toward a mathematical

\section{(2015) 1365}

25 (2015) 1887

25 (2015) 2349

25 (2015) 2217

25 (2015) 1519

25 (2015) 395

25 (2015) 2417 
theory of Keller-Segel models of pattern formation in biological tissues

Bellouquid, A., see Bellomo

Bendahmane, M., see Andreianov

Berestycki, H., Coulon, A.C., Roquejoffre, J.-M. \& Rossi, L., The effect of a line with nonlocal diffusion on Fisher-KPP propagation

Bhatt, V., see Mittal

Blass, T., see Agostiniani

Bonaldi, F., Geymonat, G. \& Krasucki, F., Modeling of smart materials with thermal effects: Dynamic and quasi-static evolution

Borzì, A. \& Wongkaew, S., Modeling and control through leadership of a refined flocking system

Borzì, A., see Wongkaew

Brauner, C.-M., see $\mathrm{Hu}$

Brezzi, F., see Bellomo

Briane, M. \& Francfort, G. A., Loss of ellipticity through homogenization in linear elasticity

Brix, K., Hafizogullari, Y. \& Platen, A., Solving the Monge-Ampère equations for the inverse reflector problem

Bru, J.-B. \& de Siqueira Pedra, W., From the second law of thermodynamics to AC-conductivity measures of interacting fermions in disordered media

Bufford, L., Davoli, E. \& Fonseca, I., Multiscale homogenization in Kirchhoff's nonlinear plate theory

Burman, E., Error estimates for forward Euler shock capturing finite element approximations of the one-dimensional Burgers' equation

Cáceres, M. J., see Chevallier

Caglioti, E., Golse, F. \& Iacobelli, M., A gradient flow approach to quantization of measures
25 (2015) 1663

25 (2015) 1663

25 (2015) 959

25 (2015) 2519

25 (2015) 2319

25 (2015) 1

25 (2015) 2633

25 (2015) 255

25 (2015) 565

25 (2015) 685

25 (2015) 395

25 (2015) 905

25 (2015) 803

25 (2015) 2587

25 (2015) 1765

25 (2015) 2015

25 (2015) 2669

25 (2015) 1845
Cai, W. L., Jabin, P.-E. \& Liu, H. L., Timeasymptotic convergence rates towards the discrete evolutionary stable distribution

Calderer, M. C. \& Chen, R. M., Long-time existence of classical solutions to a one-dimensional swelling gel

Calderer, R., Zhu, L., Gibson, R. \& Masud, A., Residual-based turbulence models and arbitrary LagrangianEulerian framework for free surface flows

Caponigro, M., Fornasier, M., Piccoli, B. \& Trélat, E., Sparse stabilization and control of alignment models

Caponigro, M., see Wongkaew

Cazeaux, P. \& Grandmont, C., Homogenization of a multiscale viscoelastic model with nonlocal damping, application to the human lungs

Chan, A. \& Conti, S., Energy scaling and branched microstructures in a model for shapememory alloys with $\mathrm{SO}(2)$ invariance

Chaplain, M., Ptashnyk, M. \& Sturrock, M., Hopf bifurcation in a gene regulatory network model: Molecular movement causes oscillations

Chen, R. M., see Calderer

Chevallier, J., Cáceres, M. J., Doumic, M. \& Reynaud-Bouret, P., Microscopic approach of a time elapsed neural model

Colasuonno, F., see Agnelli

Conti, S., see Chan

Coulon, A.-C., see Berestycki

Crooks, E., see Zhang

Crooks, E., see Zhang

Dahmen, W., see Deolmi

Danbolt, N. C., see Raynaud
25 (2015) 1589

25 (2015) 165

25 (2015) 2287

25 (2015) 521

25 (2015) 565

25 (2015) 1125

25 (2015) 1091

25 (2015) 1179

25 (2015) 165

25 (2015) 2669

25 (2015) 109

25 (2015) 1091

25 (2015) 2519

25 (2015) 747

25 (2015) 839

25 (2015) 1257

25 (2015) 195 
d'Avenia, P., Siciliano, G. \& Squassina, M., On fractional Choquard equations

Davoli, E., see Bufford

Degond, P. \& Navoret, L., A multi-layer model for self-propelled disks interacting through alignment and volume exclusion

Degond, P. \& Yu, H., Selforganized hydrodynamics in an annular domain: Modal analysis and nonlinear effects

Dellacherie, S., see Penel

Deolmi, G., Dahmen, W. \& Müller, S., Effective boundary conditions for compressible flows over rough boundaries

de Siqueira Pedra, W., see Bru

Després, B., see Penel

Donatelli, D., Feireisl, E. \& Novotný, A., Scale analysis of a hydrodynamic model of plasma

Doumic, M., see Chevallier

Duan, B., see Bae

Duan, R., Liu, Q. \& Zhu, C., Darcy's law and diffusion for a two-fluid EulerMaxwell system with dissipation

Evje, S. \& Wen, H., On the large time behavior of the compressible gas-liquid drift-flux model with slip

Farshbaf-Shaker, M. H. \& Heinemann, C., A phase field approach for optimal boundary control of damage processes in twodimensional viscoelastic media

Faure, S. \& Maury, B., Crowd motion from the granular standpoint

Feireisl, E., see Donatelli

Fermo, L. \& Tosin, A., A fully-discrete-state kinetic theory approach to traffic flow on road networks

Finkelshtein, D., Kondratiev, Y., Kozitsky, Y. \& Kutoviy, O., The statistical dynamics of a spatial logistic model and the related kinetic equation
Fletcher, A. G., Murray, P. J. \& Maini, P. K. Multiscale modelling of intestinal crypt organization and carcinogenesis

Fonseca, I., see Bufford

Fornasier, M., see Caponigro

Francfort, G. A., see Briane

25 (2015) 2439

Ganis, B., see Girault

Geymonat, G., see Bonaldi

Gibelli, L., see Bellomo

Gibson, R., see Calderer

Girault, V., Wheeler, M. F., Ganis, B. \& Mear, M. E., A lubrication fracture model in a poro-elastic medium

Gohari, S. M. I., see Bazilevs

25 (2015) 1257

25 (2015) 2587

25 (2015) 1045

25 (2015) 371

25 (2015) 2669

25 (2015) 2721

Golse, F., see Caglioti

Grandmont, C., see Cazeaux

Guillod, J. \& Wittwer, P., Asymptotic behaviour of solutions to the stationary Navier-Stokes equations in two-dimensional exterior domains with zero velocity at infinity

Ha, S.-Y., see Bae

Hafizogullari, Y., see Brix

Hattori, H., see Takizawa

Heinemann, C., see Farshbaf-Shaker

Herrero, M. A. \& Soler, J., Cooperation, competition, organization: The dynamics of interacting living populations

Herty, M. \& Ringhofer, C., Large time behavior of averaged kinetic models on networks

Herty, M., see Michailidis

25 (2015) 2749

25 (2015) 463

25 (2015) 371

25 (2015) 423

Hilhorst, D., Kampmann, J., Nguyen, T. N. \& van der Zee, K. G., Formal asymptotic limit of a diffuse-interface tumorgrowth model

$\mathrm{Hu}$, L., Brauner, C.-M., Shen, J. \& Sivashinsky, G. I., Modeling and simulation of fingering pattern formation in a combustion model

Hughes, T. J. R., see Beirão da Veiga

25 (2015) 343

Iacobelli, M., see Caglioti
25 (2015) 2563

25 (2015) 1765

25 (2015) 521

25 (2015) 905

25 (2015) 587

25 (2015) 2633

25 (2015) 2417

25 (2015) 2287

25 (2015) 587

25 (2015) 2349

25 (2015) 1845

25 (2015) 1125

25 (2015) 229

25 (2015) 1299

25 (2015) 803

25 (2015) 2377

25 (2015) 2749

25 (2015) 2407

25 (2015) 875

25 (2015) 283

25 (2015) 1011

25 (2015) 685

25 (2015) 1519

25 (2015) 1845 
Iyer, G., Xu, X. \& Zarnescu, A. D., Dynamic cubic instability in a 2D Qtensor model for liquid crystals

Jabin, P.-E., see Cai

Jiang, Y., Shu, C.-W. \& Zhang, M., High-order finite difference WENO schemes with positivitypreserving limiter for correlated random walk with density-dependent turning rates

Jüngel, A., Negulescu, C. \& Shpartko, P., Bounded weak solutions to a matrix drift-diffusion model for spin-coherent electron transport in semiconductors

Kampmann, J., see Hilhorst

Kang, M.-J. \& Vasseur, A., Asymptotic analysis of Vlasov-type equations under strong local alignment regime

Karper, T. K., Mellet, A. \& Trivisa, K., Hydrodynamic limit of the kinetic Cucker-Smale flocking model

Kawashima, S., see Xu

Kiendl, J., see Beirão da Veiga

Kim, Y., see Bae

Kitavtsev, G., Luckhaus, S. \& Rüland, A., Surface energies arising in microscopic modeling of martensitic transformations

Knopoff, D., see Agnelli

Kondratiev, Y., see Finkelshtein

Korobenko, A., see Bazilevs

Kotschote, M. \& Zacher, R., Strong solutions in the dynamical theory of compressible fluid mixtures

Koumatos, K., see Agostiniani

Kozitsky, Y., see Finkelshtein

Krasucki, F., see Bonaldi

Kuraishi, T., see Takizawa

Kutoviy, O., see Finkelshtein

Lai, J. C. S., see Tian

Lanthaler, S. \& Mishra, S., Computation of measurevalued solutions for the incompressible Euler equations

25 (2015) 1477 25 (2015) 1589

25 (2015) 1553

25 (2015) 929

25 (2015) 1011

25 (2015) 2153

25 (2015) 131

25 (2015) 1813

25 (2015) 1519

25 (2015) 1299

25 (2015) 647

25 (2015) 109

25 (2015) 343

25 (2015) 2349

25 (2015) 1217

25 (2015) 1

25 (2015) 343

25 (2015) 2633

25 (2015) 2227

25 (2015) 343

25 (2015) 2257
Lassas, M., Salo, M. \& Tzou, L., Inverse problems and invisibility cloaking for FEM models and resistor networks

Lee, S.-H., see Bae

Lehre, K. P., see Raynaud

Li, T., Suen, A., Winkler, M. \& Xue, C., Global small-data solutions of a two-dimensional chemotaxis system with rotational flux terms

Lim, H., see Bae

Liu, H. L., see Cai

Liu, Q., see Duan

Lovadina, C., see Beirão da Veiga

Luckhaus, S., see Kitavtsev

Maini, P. K., see Fletcher

Mantič, V., see Roubíček

Masud, A., see Calderer

Maury, B., see Faure

Mear, M. E., see Girault

Mei, S., see Takizawa

Mellet, A., see Karper

Michailidis, L., Herty, M. \& Ziegler, M., Kinetic partfeeding models for assembly lines in automotive industries

Mishra, S., see Lanthaler

Mittal, S., Bhatt, V. \& Srinath, D. N., Aerodynamic shape optimization using stabilized finite element method

Mochizuki, H., see Takizawa

Montel, K., see Takizawa

Mora, D., Rivera, G. \& Rodríguez, R., A virtual element method for the Steklov eigenvalue problem

Müller, S., see Deolmi

Murray, P. J., see Fletcher

Navoret, L., see Degond

Negri, M. \& Toader, R., Scaling in fracture mechanics by Bažant law: From finite to linearized elasticity

Negulescu, C., see Jüngel
25 (2015) 2043

25 (2015) 309

25 (2015) 1299

25 (2015) 195

25 (2015) 721

25 (2015) 1299

25 (2015) 1589

25 (2015) 2089

25 (2015) 1519

25 (2015) 647

25 (2015) 2563

25 (2015) 1337

25 (2015) 2287

25 (2015) 463

25 (2015) 587

25 (2015) 2377

25 (2015) 131

25 (2015) 283

25 (2015) 2043

25 (2015) 2319

25 (2015) 2377

25 (2015) 2377

25 (2015) 1421

25 (2015) 1257

25 (2015) 2563

25 (2015) 2439

25 (2015) 1389

25 (2015) 929 
Nguyen, H.-M. \& Nguyen, L. V., Generalized impedance boundary conditions for strongly absorbing obstacle: The full wave equation

Nguyen, L. V., see Nguyen

Nguyen, T. N., see Hilhorst

Niiranen, J., see Beirão da Veiga

Nordaas, M., see Raynaud

Novotný, A., see Donatelli

Orlando, A., see Zhang

Orlando, A., see Zhang

Pacheco, J. M., see Vasconcelos

Pal, A., see Bazilevs

Pan, L., see Takizawa

Panagiotopoulos, C. G., see Roubíček

Penel, Y., Dellacherie, S. \& Després, B., Coupling strategies for compressible-low Mach number flows

Penta, R., Ambrosi, D. \& Quarteroni, A., Multiscale homogenization for fluid and drug transport in vascularized malignant tissues

Perthame, B., Quiñinao, C. \& Touboul, J., Competition and boundary formation in heterogeneous media: Application to neuronal differentiation

Piccoli, B., see Caponigro

Platen, A., see Brix

Ptashnyk, M., see Chaplain

Pulvirenti, M., see Aoki

Quarteroni, A., see Andreianov

Quarteroni, A., see Penta

Quiñinao, C., see Perthame

Raynaud, X., Nordaas, M., Lehre, K. P. \& Danbolt, N. C., Well-posedness of a model equation for neurotransmitter diffusion with reactive boundaries

Reali, A., see Beirão da Veiga

Reynaud-Bouret, P., see Chevallier

Ringhofer, C., see Herty

Rivera, G., see Mora

Rodríguez, R., see Mora

Roquejoffre, J.-M., see Berestycki

Rossi, L., see Berestycki
25 (2015) 1927

25 (2015) 1927

25 (2015) 1011

25 (2015) 1519

25 (2015) 195

25 (2015) 371

25 (2015) 747

25 (2015) 839

25 (2015) 2503

25 (2015) 2349

25 (2015) 2377

25 (2015) 1337

25 (2015) 1045

25 (2015) 79

25 (2015) 2477

25 (2015) 521

25 (2015) 803

25 (2015) 1179

25 (2015) 995

25 (2015) 959

25 (2015) 79

25 (2015) 2477

25 (2015) 195

25 (2015) 1519

25 (2015) 2669

25 (2015) 875

25 (2015) 1421

25 (2015) 1421

25 (2015) 2519

25 (2015) 2519
Roubíček, T., Panagiotopoulos, C. G. \& Mantič, V., Local-solution approach to quasistatic rate-independent mixedmode delamination

Ruiz-Baier, R., see Andreianov

Rüland, A., see Kitavtsev

Salo, M., see Lassas

Santos, F. C., see Vasconcelos

Sarkar, S., see Bazilevs

Schadschneider, A., see Arita

Schötzau, D. \& Schwab, C., Exponential convergence for $h p$-version and spectral finite element methods for elliptic problems in polyhedra

Schwab, C., see Schötzau

Shen, J., see $\mathrm{Hu}$

Shpartko, P., see Jüngel

Shu, C.-W., see Jiang

Siciliano, G., see d'Avenia

Simonella, S., see Aoki

Sivashinsky, G. I., see $\mathrm{Hu}$

Soler, J., see Herrero

Speleers, H., see Beirão da Veiga

Squassina, M., see d'Avenia Srinath, D. N., see Mittal

Stratis, I. G., see Barbatis

Sturrock, M., see Chaplain

Suen, A., see $\mathrm{Li}$

Takizawa, K., Tezduyar, T. E. \& Kuraishi, T., Multiscale space-time methods for thermo-fluid analysis of a ground vehicle and its tires

Takizawa, K., Tezduyar, T. E., Mochizuki, H., Hattori, H., Mei, S., Pan, L. \& Montel, K., Spacetime VMS method for flow computations with slip interfaces (ST-SI)

Takizawa, K., see Bazilevs

Tao, Y., see Bellomo

Tezduyar, T. E., see Bazilevs

Tezduyar, T. E., see Takizawa

Tezduyar, T. E., see Takizawa

Tian, F.-B., Wang, Y., Young, J. \& Lai, J. C. S., An FSI solution technique based on the DSD/SST
25 (2015) 1337

25 (2015) 959

25 (2015) 647

25 (2015) 309

25 (2015) 2503

25 (2015) 2349

25 (2015) 401

25 (2015) 1617

25 (2015) 1617

25 (2015) 685

25 (2015) 929

25 (2015) 1553

25 (2015) 1447

25 (2015) 995

25 (2015) 685

25 (2015) 2407

25 (2015) 1519

25 (2015) 1447

25 (2015) 2319

25 (2015) 1365

25 (2015) 1179

25 (2015) 721

25 (2015) 2227

25 (2015) 2377

25 (2015) 2217

25 (2015) 1663

25 (2015) 2217

25 (2015) 2227

25 (2015) 2377 
method and its applications

Toader, R., see Negri

Toscani, G., see Bassetti

Tosin, A., see Fermo

Touboul, J., see Perthame

Trélat, E., see Caponigro

Trivisa, K., see Karper

Tsuji, T., see Aoki

Turanova, O., On a model of a population with variable motility

Tzou, L., see Lassas

Vasconcelos, V. V., Santos, F. C. \& Pacheco, J. M., Cooperation dynamics of polycentric climate governance

Vasseur, A., see Kang

van der Zee, K. G., see Hilhorst

Wang, Y., see Tian

Wen, H., see Evje

Wheeler, M. F., see Girault

Winkler, M., see Bellomo

Winkler, M., see $\mathrm{Li}$

Wittwer, P., see Guillod

Wongkaew, S., Caponigro, M. \& Borzì, A., On the control through leadership of the HegselmannKrause opinion formation model

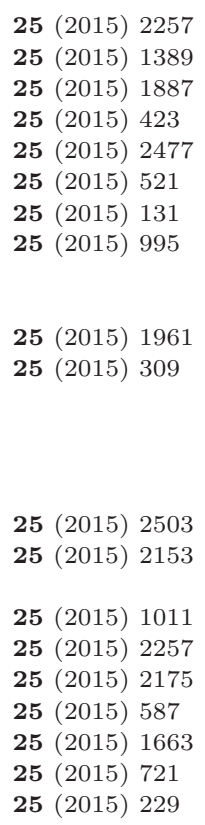

25 (2015) 2257

25 (2015) 1389

25 (2015) 1887

25 (2015) 423

25 (2015) 2477

25 (2015) 521

25 (2015) 131

25 (2015) 995

25 (2015) 1961

25 (2015) 309

25 (2015) 2503

25 (2015) 2153

25 (2015) 1011

25 (2015) 2257

25 (2015) 2175

25 (2015) 587

25 (2015) 1663

25 (2015) 721

25 (2015) 229

25 (2015) 565
Wongkaew, S., see Borzì

Xie, C., see Bae

Xu, J. \& Kawashima, S., The optimal decay estimates on the framework of Besov spaces for the Euler-Poisson two-fluid system

$\mathrm{Xu}, \mathrm{X}$., see Iyer

Xue, C., see $\mathrm{Li}$

Yan, J., see Bazilevs

Yannacopoulos, A. N., see Barbatis

Yoo, J., see Bae

Young, J., see Tian

Yu, H., see Degond

Zacher, R., see Kotschote

Zarnescu, A. D., see Iyer

Zhang, K., Orlando, A. \& Crooks, E., Compensated convexity and Hausdorff stable extraction of intersections for smooth manifolds

Zhang, K., Orlando, A. \& Crooks, E., Compensated convexity and Hausdorff stable geometric singularity extractions

Zhang, M., see Jiang

Zhu, C., see Duan

Zhu, L., see Calderer

Ziegler, M., see Michailidis
25 (2015) 255

25 (2015) 2721

25 (2015) 1813

25 (2015) 1477

25 (2015) 721

25 (2015) 2349

25 (2015) 1365

25 (2015) 1299

25 (2015) 2257

25 (2015) 495

25 (2015) 1217

25 (2015) 1477

25 (2015) 839

25 (2015) 747

25 (2015) 1553

25 (2015) 2089

25 (2015) 2287

25 (2015) 283 